\title{
Direccionamiento Estratégico en Servicios Gerontológicos. La Percepción de los Directivos en Tabasco
}

\section{Strategic Direction in Gerontological Services. The Perception of Managers in Tabasco}

\author{
Raquel Adriana Rodríguez Santos*, Heberto Romeo Priego Álvarez**, \\ Santiago López Solorsano***, Diana Caldelas García****
}

* Cirujano. Estudiante de la Maestría en Salud Pública. División Académica de Ciencias de la Salud. Universidad Juárez Autónoma de Tabasco. Villahermosa, Tabasco. ORCID: https://orcid.org/0000-00019901-6931.

** Doctor en Ciencias de la Salud. Profesor investigador de la División Académica de Ciencias de la Salud de la Universidad Juárez Autónoma de Tabasco. Villahermosa, Tabasco. ORCID: https://orcid.org/00000001-9217-5702

*** Estudiante de medicina en verano científico. División Académica de Ciencias de la Salud. Universidad Juárez Autónoma de Tabasco. Villahermosa, Tabasco. ORCID: https://orcid.org/0000-0001-6083-8583.

**** Estudiante de medicina en verano científico. División Académica de Ciencias de la Salud. Universidad Juárez Autónoma de Tabasco. Villahermosa, Tabasco. ORCID: https://orcid.org/0000-0002-7658-8045.

Correo para recibir correspondencia: heberto_priego@hotmail.com 
DIRECCIONAMIENTO ESTRATÉGICO EN SERVICIOS GERONTOLÓGICOS. LA PERCEPCIÓN DE LOS DIRECTIVOS EN TABASCO.

\section{RESUMEN}

Los servicios gerontológicos han demostrado ser una alternativa adecuada para garantizar la calidad de vida del adulto mayor, por lo que es importante conocer los elementos que intervienen en su gestión.

OBJETIVO: Describir el direccionamiento estratégico en los servicios gerontológicos desde la perspectiva de los directivos tabasqueños.

MATERIALES Y MÉTODO: Se realizó un estudio cualitativo, descriptivo, de corte transversal que abarcó un periodo de dos meses comprendido de julio a agosto de 2020. La población en estudio fueron cuatro directivos clave de instituciones que prestan servicios gerontológicos. La obtención de los datos se efectuó a través de entrevistas semiestructuradas. El procesamiento de la información se realizó mediante el análisis de contenido, utilizando el software ATLAS.ti versión 8 y el procesador de textos Microsoft Word.

RESULTADOS: El punto de convergencia entre los objetivos establecidos por los directivos es, priorizar la funcionalidad, mejorar la calidad de vida y preservar la integridad física, psicológica y social del adulto mayor. Asimismo, es necesario Constituir una estructura clara, que permita establecer niveles jerárquicos, distribuir y evitar la duplicidad de funciones, y optimizar los recursos financieros, humanos, materiales y tecnológicos.

CONCLUSIONES: El estudio realizado permitió la identificación de ciertos elementos que contribuyen al funcionamiento de las instituciones gerontológicas en Tabasco, en particular, el reconocimiento del objetivo fundamental de la atención integral del adulto mayor, al enfoque en los procesos directivos y al pragmatismo de los centros privados.

PALABRAS CLAVE: Directivos. Servicios gerontológicos. Estrategias. Instituciones. Envejecimiento. 
DIRECCIONAMIENTO ESTRATÉGICO EN SERVICIOS GERONTOLÓGICOS. LA PERCEPCIÓN DE LOS DIRECTIVOS EN TABASCO.

\section{ABSTRACT}

Gerontological services have proven to be an adequate alternative to guarantee the quality of life of the elderly, so it is important to know the elements that intervene in their management.

OBJECTIVE: To describe the strategic direction in gerontological services from the perspective of the Managers from Tabasco.

MATERIALS AND METHOD: From July to August 2020, a two month qualitative, descriptive, cross-sectional study was carried out. The study population was four key directors of institutions that provide gerontological services. The data was obtained through semi-structured interviews. The information processing was carried out through content analysis, using the ATLAS.ti version 8 software and the Microsoft Word processor.

RESULTS: The points of convergence between the objectives established by the Managers are: to prioritize functionality, improve the quality of life and preserve the physical, psychological and social integrity of the elderly. Likewise, it is necessary to constitute a clear structure that allows establishing hierarchical levels, distributing and avoiding duplication of functions, and optimizing financial, human, material and technological resources.

CONCLUSIONS: This study allowed the identification of certain elements which contribute to the functioning of gerontological institutions in Tabasco; in particular, the recognition of the fundamental objective of comprehensive care for the elderly, the focus on directive processes and the pragmatism of the private centers.

KEY WORDS: Executives. Gerontological services. Strategies. Institutions. Aging.

\section{INTRODUCCIÓN}

El envejecimiento poblacional, es uno de los indicadores del desarrollo de los países. En el estado mexicano de Tabasco, gracias a los avances tecnológicos de la medicina y de las estrategias en materia de salud pública, la esperanza de vida ha aumentado a lo largo de los años, siendo actualmente de 75 años (Consejo Nacional de Población [CONAPO], 2019). Esto significa que 
DIRECCIONAMIENTO ESTRATÉGICO EN SERVICIOS GERONTOLÓGICOS. LA PERCEPCIÓN DE LOS DIRECTIVOS EN TABASCO.

las personas están viviendo más años, por lo que se crea la necesidad de políticas de asistencia social adaptadas a las características de la población envejecida y el desarrollo de modelos de atención gerontológica.

De acuerdo con el Directorio Estadístico Nacional de Unidades Económicas (DENUE) del Instituto Nacional de Estadística, Geografía e Historia (INEGI), "en México existen 819 asilos y otras residencias para el cuidado del anciano, de las cuales, un $85 \%$ corresponden al sector privado $y$ 15\% al sector público" (Citado por PROFECO, 2018).

Los servicios gerontológicos han demostrado ser una alternativa adecuada para garantizar la participación social, mantener la autonomía, proteger los derechos humanos fundamentales y mejorar la calidad de vida de los adultos mayores. Ante esto, es importante conocer los elementos que intervienen en la conducción de las organizaciones de atención gerontológica.

El direccionamiento estratégico es un proceso mediante el cual se realizan planeaciones estratégicas y de objetivos que definen la orientación que tomara un producto o servicio en el mercado (Trujillo, 2006) y permite a los directivos de la organización cumplir las metas establecidas, canalizando recursos y acciones en el mismo sentido (Gutiérrez, 2016).

Existen diferentes organismos cuyas metas son el bienestar de la población envejecida durante su estancia, ya sea a corto o largo plazo (PROFECO, 2018; Góngora, J. 2018), cuyos planteamientos estratégicos, están adaptados a las necesidades del adulto mayor y que se han ido reestructurando en beneficio de estos usuarios con el paso del tiempo (INAPAM, 2012; Secretaría de Bienestar, 2019).

Este estudio tiene como objetivo describir el direccionamiento estratégico en los servicios gerontológicos en Tabasco desde la perspectiva de sus directivos. Para esto, se identifican los objetivos fundamentales de las instituciones y se describen los procesos para definirlos y lograrlos; así como la estructura organizacional, la identificación de fortalezas y debilidades de las organizaciones, y la sensibilidad a las expectativas y necesidades de los usuarios.

\section{MATERIAL Y MÉTODO}

Se realizó un estudio cualitativo, descriptivo, de corte transversal cuya recolección de datos abarcó los meses de julio a agosto de 2020. La población en estudio fueron directivos de instituciones de atención gerontológica. Se definieron los perfiles de los participantes, 
DIRECCIONAMIENTO ESTRATÉGICO EN SERVICIOS GERONTOLÓGICOS. LA PERCEPCIÓN DE LOS DIRECTIVOS EN TABASCO.

considerando a todo graduado del campo de las ciencias de la salud o sociales que tuviera un cargo directivo en una institución de atención gerontológica pública y/o privada, cuya antigüedad en el puesto hubiera sido mayor a seis meses; y que aceptara participar en el estudio, previo consentimiento informado.

Mediante un muestreo de tipo opinático, se seleccionaron 4 informantes clave a quienes se les realizaron entrevistas semiestructuradas. El guion de entrevista diseñado contiene 15 ítems agrupados en cuatro ejes temáticos: a) características del direccionamiento estratégico de la institución, b) percepción del desempeño de la institución, c) percepción del desempeño de los servicios estatales de atención al adulto mayor, y d) percepción de logros y desafíos en la atención de las personas mayores en Tabasco.

Para propósitos de este estudio se consideraron las cuatro dimensiones que corresponden al análisis del direccionamiento estratégico, a partir de las cuales se desglosan las categorías de análisis (ver Tabla 1).

\section{Tabla 1}

Categorías de análisis de los directivos entrevistados

\begin{tabular}{|c|c|c|}
\hline Categoría & Definición conceptual & Ítem \\
\hline $\begin{array}{l}\text { Objetivos } \\
\text { organizacionales }\end{array}$ & $\begin{array}{l}\text { Descripción de los propósitos } \\
\text { organizacionales y estrategias para } \\
\text { definirlos y priorizarlos. }\end{array}$ & $\begin{array}{l}\text { ¿Cuáles son los objetivos } \\
\text { fundamentales en la atención } \\
\text { gerontológica de la institución que } \\
\text { dirigió? } \\
\text { ¿De qué forma define objetivos y los } \\
\text { procesos para alcanzarlos? }\end{array}$ \\
\hline $\begin{array}{l}\text { Estructura } \\
\text { organizacional }\end{array}$ & $\begin{array}{l}\text { Descripción de la distribución los } \\
\text { recursos materiales, financieros, } \\
\text { humanos y tecnológicos de la } \\
\text { institución de acuerdo con sus } \\
\text { requerimientos. }\end{array}$ & $\begin{array}{l}\text { ¿Cómo estaba organizada la } \\
\text { institución? }\end{array}$ \\
\hline $\begin{array}{l}\text { Identificación de } \\
\text { fortalezas y debilidades } \\
\text { de la organización }\end{array}$ & $\begin{array}{l}\text { Capacidad analítica y directiva en la } \\
\text { identificación del ambiente interno } \\
\text { (fortalezas y debilidades) de la } \\
\text { organización. }\end{array}$ & $\begin{array}{l}\text { ¿Cuáles son las fortalezas y } \\
\text { debilidades que veía en la institución? }\end{array}$ \\
\hline $\begin{array}{l}\text { Sensibilidad a las } \\
\text { expectativas y } \\
\text { demandas de los } \\
\text { usuarios }\end{array}$ & $\begin{array}{l}\text { Estrategias para identificar las } \\
\text { necesidades y prioridades de } \\
\text { atención gerontológica de los } \\
\text { usuarios. }\end{array}$ & $\begin{array}{l}\text { ¿De qué forma identificaba las } \\
\text { expectativas y necesidades sentidas } \\
\text { por las personas mayores? }\end{array}$ \\
\hline
\end{tabular}

Fuente: Elaboración propia. 
DIRECCIONAMIENTO ESTRATÉGICO EN SERVICIOS GERONTOLÓGICOS. LA PERCEPCIÓN DE LOS DIRECTIVOS EN TABASCO.

Debido a la actual alerta sanitaria por la COVID-19, las entrevistas se realizaron mediante videollamadas realizadas a través de plataformas digitales y fueron grabadas en audio, previa autorización de los participantes para su transcripción posterior. La duración promedio de cada entrevista fue de 60 minutos.

El procesamiento de los datos se realizó mediante el análisis de contenido, en cuya etapa inicial se identificaron y codificaron las categorías a analizar. Posteriormente se realizó el ordenamiento de los discursos narrativos en cada categoría y se seleccionaron las frases representativas para su caracterización. La sistematización de la información se realizó en el software ATLAS.ti versión 8 (The Qualitative Data Analysis \& Research y el procesador de textos Microsoft Word.

\section{RESULTADOS Y DISCUSIÓN}

Las características sociodemográficas de los informantes (directivos de servicios gerontológicos) se detallan en la Tabla 2.

\section{Tabla 2}

Características sociodemográficas de los directivos entrevistados

\begin{tabular}{|c|c|c|c|c|c|}
\hline $\begin{array}{l}\text { Folio de } \\
\text { identificación }\end{array}$ & Edad & Sexo & Formación & Cargos directivos & $\begin{array}{l}\text { Antigüedad en } \\
\text { el cargo }\end{array}$ \\
\hline SM-D01 & 50 & Mujer & $\begin{array}{l}\text { Médico Cirujano con } \\
\text { estudios de posgrado en } \\
\text { Geriatría, Demencias y } \\
\text { Enfermedades } \\
\text { Neurodegenerativas. }\end{array}$ & $\begin{array}{l}\text { Directora de un } \\
\text { centro de día del } \\
\text { sector privado }\end{array}$ & $\begin{array}{c}14 \text { años, } 8 \\
\text { meses }\end{array}$ \\
\hline SM-D02 & 36 & Mujer & $\begin{array}{c}\text { Médico Cirujano con } \\
\text { estudios de posgrado en } \\
\text { Geriatría }\end{array}$ & $\begin{array}{l}\text { Subdirectora de } \\
\text { una residencia } \\
\text { geriátrica del } \\
\text { sector público }\end{array}$ & 6 meses \\
\hline SM-D03 & 46 & Mujer & $\begin{array}{c}\text { Médico Cirujano con } \\
\text { estudios de posgrado en } \\
\text { Gerontología Social, } \\
\text { Administración y Políticas } \\
\text { Públicas. }\end{array}$ & $\begin{array}{l}\text { Directora de un } \\
\text { centro } \\
\text { gerontológico del } \\
\text { sector público }\end{array}$ & $\begin{array}{c}3 \text { años y } 6 \\
\text { meses }\end{array}$ \\
\hline SM-D04 & 65 & Hombre & $\begin{array}{c}\text { Médico Cirujano con } \\
\text { especialidad en } \\
\text { Traumatología y Ortopedia } \\
\text { y posgrado en Gerontología } \\
\text { Social. }\end{array}$ & $\begin{array}{l}\text { Delegado federal } \\
\text { del Instituto } \\
\text { Nacional de las } \\
\text { Personas Adultas } \\
\text { Mayores }\end{array}$ & 34 años \\
\hline
\end{tabular}

Fuente: Elaboración propia. 
DIRECCIONAMIENTO ESTRATÉGICO EN SERVICIOS GERONTOLÓGICOS. LA PERCEPCIÓN DE LOS DIRECTIVOS EN TABASCO.

Se identificó una red de interrelaciones del contenido de las categorías y discursos representativos de los directivos de instituciones gerontológicas (ver Figura 1), mediante las cuales se abordaron las categorías de análisis.

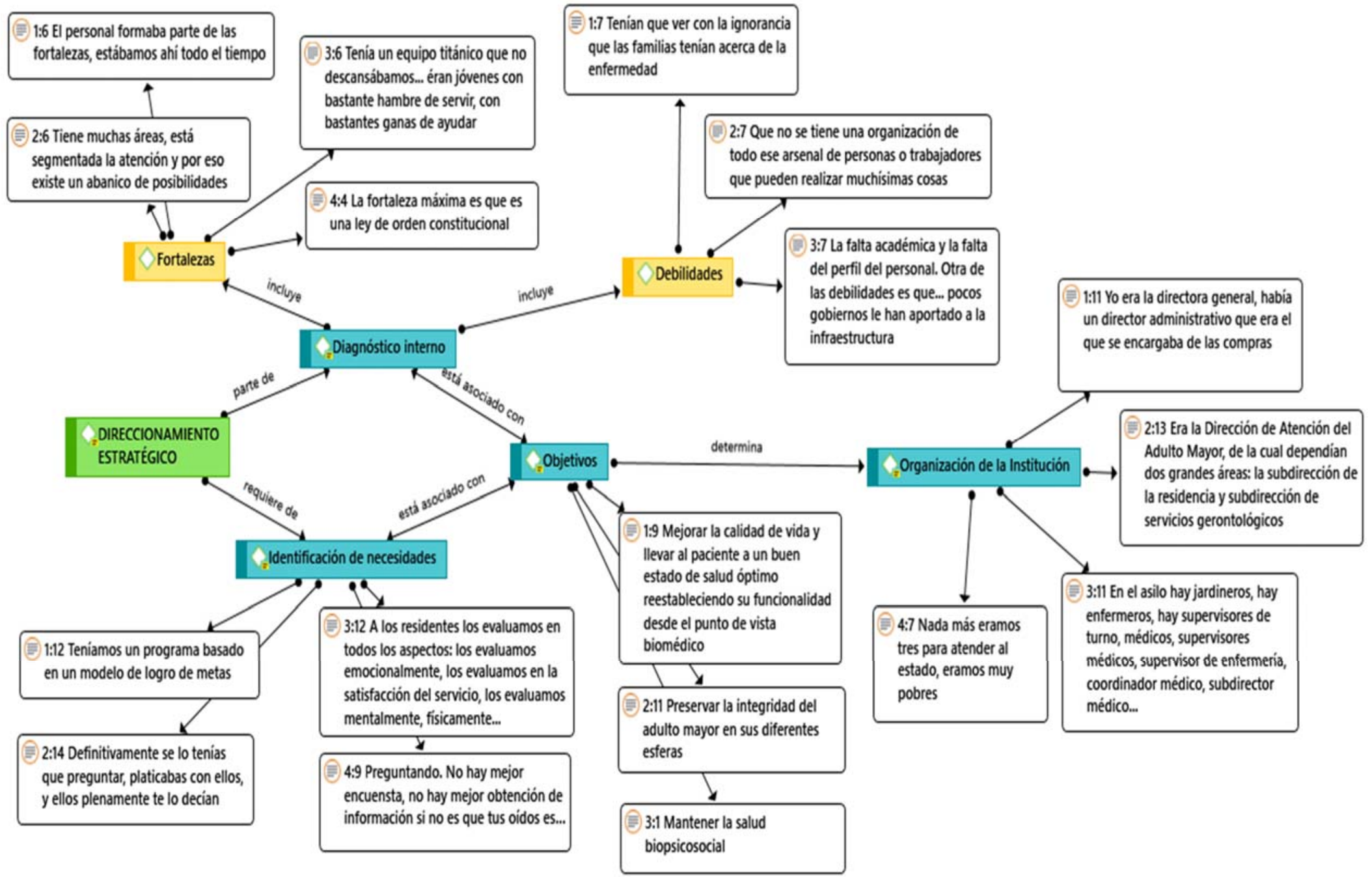

Figura 1. Red de interrelaciones del contenido de las categorías y discursos representativos de los directivos de instituciones gerontológicas.

Fuente: Elaboración propia a partir del análisis de los discursos emitidos por los directivos de instituciones gerontológicas entrevistados.

RODRÍGUEZ-SANTOS R. A., PRIEGO-ÁLVAREZ H. R., LÓPEZ-SOLORSANO S., CALDELAS-GARCÍA D.

HITOS DE CIENCIAS ECONOMICO ADMINISTRATIVAS ENERO-ABRIL 2021. Año 27, Número 77. Págs. 94-108 
DIRECCIONAMIENTO ESTRATÉGICO EN SERVICIOS GERONTOLÓGICOS. LA PERCEPCIÓN DE LOS DIRECTIVOS EN TABASCO.

\section{Objetivos de atención gerontológica en las instituciones y procesos para alcanzarlos.}

La definición de objetivos es un elemento clave en la gestión de las organizaciones. Este proceso gerencial es dinámico y tiene como propósito fundamental responder a las demandas internas y externas de la organización; por lo que ocurre de manera particular en cada institución de acuerdo con su misión y visión, los recursos disponibles y las características del entorno. Para las organizaciones de atención gerontológica, los objetivos suelen corresponderse con el modelo de atención al que pertenecen.

Un punto de convergencia observado fue el objetivo organizacional; en cuyo caso los directivos coincidieron con que: "priorizar la funcionalidad, mejorar la calidad de vida y preservar su integridad física, psicológica y social", es el propósito fundamental de las instituciones de atención gerontológica (ver Tabla 3).

\section{Tabla 3}

Objetivos de atención gerontológica para cada institución

\begin{tabular}{|c|c|}
\hline Categoría & Discurso \\
\hline $\begin{array}{l}\text { Objetivos } \\
\text { fundamentales de la } \\
\text { atención gerontológica }\end{array}$ & $\begin{array}{l}\text { "... mejorar la calidad de vida y llevar al paciente a un buen estado de salud óptimo, } \\
\text { restableciendo su funcionalidad desde el punto de vista biomédico". SM-D01. } \\
\text { “...era preservar la integridad del adulto mayor en sus diferentes esferas, ya sea en } \\
\text { el ámbito de la salud, el ámbito de actividad física, de la cuestión psicológica, y } \\
\text { mantener la integridad social dentro y fuera de la institución”. SM-D02. } \\
\text { “...mantener la salud biopsicosocial, o sea, no solamente era tenerlos físicamente } \\
\text { bien sino mentalmente bien y también lúdicamente bien, o sea, que se sintieran } \\
\text { bien”. SM-D03. } \\
\text { “...el interés era proteger, orientar, cuidar, velar por la vejez mexicana, esos eran } \\
\text { sus objetivos y esto a su vez, era parte de una recomendación presidencial”. SM- } \\
\text { D04. }\end{array}$ \\
\hline
\end{tabular}

Fuente: Elaboración propia.

Respecto a los procesos para definir los objetivos y lograrlos, se encontraron algunas diferencias. En el centro de día privado, se mantuvo un modelo de valoración geriátrica integral para definir un plan de trabajo terapéutico, en el que también se incluía la participación de la familia.

La valoración geriátrica integral es un proceso de diagnóstico que califica el estado físico, psicológico y social, para diseñar una estrategia preventiva, terapéutica y rehabilitadora personalizada, cuya finalidad es preservar o recobrar la funcionalidad y autonomía (Gálvez, 
DIRECCIONAMIENTO ESTRATÉGICO EN SERVICIOS GERONTOLÓGICOS. LA PERCEPCIÓN DE LOS DIRECTIVOS EN TABASCO.

2016). Este enfoque de valoración, permite definir objetivos centrados en el adulto mayor y facilita la evaluación de su cumplimiento mediante indicadores clínicos.

Para el caso de las instituciones públicas, se observó que la definición de los objetivos se llevaba a cabo mediante la integración de equipos multidisciplinarios con expertos que se encargaban de identificar las necesidades de la institución por áreas y departamentos, e integraban un plan de trabajo. Además, cabe destacar que los recursos financieros eran considerados como un factor importante para definir estos procesos y priorizarlos (ver Tabla 4).

\section{Tabla 4}

Procesos empleados para definir y alcanzar objetivos de cada institución

Categoría Discurso

Procesos para el definir y alcanzar los objetivos

\begin{abstract}
“... lo baso en la evaluación geriátrica integral, manejo escalas de valoración geriátrica y evalúo al paciente integralmente y veo donde están sus déficits $y$ planteo puntos a mejorar en ellos. Esto con la intención de que la familia incluso sepa qué hacer con ellos desde casa". SM-D01.

“...de forma integral y colaborativa. Un equipo de expertos de cada área integraba una mesa en la cual se tomaban las decisiones para poder ejercer algún plan o de alguna manera cambiarlo o retirarlo". SM-D02.

“...ir evaluando área por área sabiendo qué operatividad tenía, cuál era el gasto, qué necesidades tenían... todo el equipo de trabajo, que era mi equipo, sabíamos qué se estaba necesitando, qué área era la que necesitaba, cuánto necesitaba, y se tenía que resolver". SM-D03.

"Bien. A ver, ¿te gustaría que mi recurso mensual era de siete mil pesos? y que tenía yo que con una lupa así poner "Factura número tal para comprar papel sanitario para los baños de ahí de la delegación" "Factura número tal para comprar papelería" "Factura número tal para comprar agua para los garrafones de agua para ahí" "Factura número tal para un vale de gasolina", que solo le daban 1000 pesos al mes para hacer dos-tres salidas al sitio y todo lo demás yo lo pagaba". SM-D04.
\end{abstract}

Fuente: Elaboración propia.

Diversos estudios en América Latina ya han descritos planes y procesos de atención con base en una segmentación diferenciada, enfatizando el propósito de ofrecer servicios diferenciados a los adultos mayores (Huenchuan, 2015; García, 2013). Los participantes en este estudio afirmaron que las características y necesidades biológicas, psicológicas y sociales definen el curso de acción de los planes estratégicos. 
DIRECCIONAMIENTO ESTRATÉGICO EN SERVICIOS GERONTOLÓGICOS. LA PERCEPCIÓN DE LOS DIRECTIVOS EN TABASCO.

\section{Estructura organizacional de las instituciones de atención gerontológica}

La estructura organizacional de las instituciones de atención gerontológica es un elemento clave que contribuye al alcance de los objetivos. Constituir una estructura clara, permite establecer niveles jerárquicos, distribuir y evitar la duplicidad de funciones, y optimizar los recursos financieros, humanos, materiales y tecnológicos. Como parte del direccionamiento estratégico de las instituciones de atención gerontológica, este proceso no es estático; y deberá responder al surgimiento de nuevos y complejos escenarios para la organización.

La organización y dirección de los centros gerontológicos privados tienden al pragmatismo. En el centro de día estudiado, se contaba con dos directores: el director general; encargado de las áreas de atención médica, enfermería, psicología y nutrición; y el director administrativo; encargado del abastecimiento de insumos y coordinación de las actividades de aseo y cocina. En contraste, las instituciones públicas suelen ser más complejas. El caso más representativo de nuestro estudio estaba constituido por una dirección de atención al adulto mayor, y dos subdirecciones: la subdirección de servicios gerontológicos y la subdirección de la institución; de esta última dependían las áreas de subdirección médica (psicología, nutrición, rehabilitación y trabajo social) y subdirección administrativa (seguimiento operativo, almacén, mantenimiento y lavandería). Modelos de organización similares han sido descritos en otras instituciones a nivel nacional e internacional (Assisted Living Workgroup, 2003; INAPAM, 2012; García Santacruz, M, 2013). Si bien, existen distintos Modelos de Atención Gerontológica en nuestro país (INAPAM, 2012), se sabe que estas instituciones basan sus planes estratégicos en la atención integral al adulto mayor, por lo que la estructura organizacional se constituye de áreas y departamentos multidisciplinarios.

Las características de la organización de las instituciones descritas por los directivos participantes del estudio, se apuntan en la Tabla 5. 
DIRECCIONAMIENTO ESTRATÉGICO EN SERVICIOS GERONTOLÓGICOS. LA PERCEPCIÓN DE LOS DIRECTIVOS EN TABASCO.

\section{Tabla 5}

Organización institucional descrita por los directivos

\begin{tabular}{ll}
\hline Categoría & Discurso \\
\hline Organización de la & "Yo era la directora general, había un director administrativo que era el que se encargaba de \\
institución & compras, de materiales, de todo eso... Teníamos un área de psicología, nutrición; en el área \\
& de nutrición estaba una nutrióloga y una cocinera; en el área de psicología había dos \\
& psicólogas; en el área de enfermería tenía dos enfermeras que trabajaban con los adultos \\
& mayores. Estaba yo como médico, tenía un epidemiólogo que nos apoyaba en el área de estar \\
& pendiente de las cuestiones de infecciosas y todo eso. El personal de aseo dependía \\
& directamente del director administrativo, el personal de aseo, y todo lo que tenía que ver con \\
& cocina". SM-D01. \\
& \\
& “...había un director que además era la dirección de atención al adulto mayor, de la cual \\
& dependían dos áreas grandes, la subdirección de la residencia y subdirección de servicios \\
& gerontológicos. Las dos con diferentes temáticas y diferentes formas de tratar a un adulto \\
& mayor. Dentro de residencia, que fue mi área en específico, de la subdirección dependía la \\
& subdirección médica y la subdirección administrativa y a partir de ellos todos los demás rubros. \\
& Por ejemplo, en el área de la subdirección administrativa, dependía el área de seguimiento \\
operativo, de almacén, de mantenimiento, lavandería, etcétera, todo lo que tenía que ver con \\
ello. Y del área de la subdirección médica dependía el área de psicología, rehabilitación, \\
nutrición, trabajo social, etcétera". SM-D02.
\end{tabular}

“... hay jardineros, hay enfermeros, hay supervisores de turno que son enfermeros, hay médicos, hay supervisor de medicina, supervisor de enfermería, coordinador médico, subdirector, trabajadoras sociales, rehabilitadores, choferes, cocineros, chefs, nutriólogos, en fin, casi de la mayoría de las disciplinas en salud estamos casi completos". SM-D03.

"nada más éramos tres para atender al estado, éramos muy pobres, éramos de las instituciones federales éramos la más pobre; pero que no necesitábamos dinero nosotros para poder hacer tantas cosas. La gente nos ayudaba, la sociedad venía". SM-D04.

Fuente: Elaboración propia.

\section{Identificación de fortalezas y debilidades en las organizaciones de atención gerontológica}

La identificación de fortalezas y debilidades de la organización forma parte de las herramientas para determinar los recursos con los que responderá a los objetivos y a las situaciones emergentes a las puede enfrentarse. Conocer las características que influyen en la capacidad de respuesta organizacional, contribuye a minimizar la incertidumbre. Para precisar estos elementos, se interrogó a los directivos acerca de las fortalezas y debilidades que identificaron en el ejercicio de sus funciones al frente de la organización. 
DIRECCIONAMIENTO ESTRATÉGICO EN SERVICIOS GERONTOLÓGICOS. LA PERCEPCIÓN DE LOS DIRECTIVOS EN TABASCO.

\title{
Tabla 6
}

Fortalezas y debilidades identificadas por los directivos

Categoría Discurso

Fortalezas y debilidades

Fortalezas

de la institución

\begin{abstract}
"Siento que el personal formaba parte de las fortalezas, estábamos ahí todo el tiempo, estábamos pendientes del centro". "Puse el sistema cerrado, lo conectamos por internet y estuviéramos donde estuviéramos, yo podía supervisar y ellos también como hijos". SM-D01.

"Como fortalezas, tiene muchas áreas, está segmentada la atención y por eso existe el abanico de posibilidades para poder atender al adulto mayor desde diferentes puntos de vista, diferentes vertientes, en base a que sea una atención integral y eso es algo benéfico". SM-D02.

"Tenía un equipo titánico que no descansábamos, en la administración pasada eran jóvenes con bastante hambre de servir, con bastantes ganas de ayudar y de cambiar las cosas porque veían todo sucio. La otra de las fortalezas era de que algunas de las propuestas que yo hice para mejorar, me hicieron caso". SM-D03.
\end{abstract}

"La fortaleza máxima es que es de orden constitucional. Número dos: de que el Instituto es un organismo público, descentralizado, supeditado a la Secretaría del Bienestar". SM-D04.

Debilidades

"Las debilidades creo que tenían que ver con la ignorancia a veces que las familias acerca de la enfermedad, de las demencias específicamente. No había una tarde que no hablara un hijo para decirnos "oiga, fíjese que es que mi papá dice que hoy no le dieron de comer". SM-D01.

"Dentro de las debilidades está precisamente que no se tiene una organización de todo este arsenal de personas o trabajadores que pueden realizar muchísimas cosas o emprender muchísimas acciones que pueden hacer." SM-D02.

"De las debilidades, pues la falta académica y la falta del perfil del personal. Otra de las debilidades es de que bueno, pocos gobiernos le han aportado a la infraestructura, por eso el asilo está catastrófico, como pá caerse, las mallas perimetrales que dividen el asilo para lo demás, son mallas herrumbradas, vencidas, comidas por el tiempo." SM-D03.

Fuente: Elaboración propia.

\section{Sensibilidad ante las expectativas y necesidades de los usuarios}

El análisis del entorno de las organizaciones gerontológicas permite identificar las exigencias y expectativas de los usuarios, a fin de establecer objetivos acordes a ellas. Este proceso es un elemento que puede determinar el éxito o el fracaso de las estrategias de atención gerontológica y de los procesos para obtener buenos resultados. Por esta razón, se solicitó a los informantes en estudio, que describieran las estrategias empleadas para la identificación de expectativas y necesidades de los adultos mayores. La institución privada, optó por utilizar un programa basado en un modelo de logro de metas que resultaba de la valoración geriátrica integral, remarcando la importancia de involucrar al adulto mayor y su familia, como participantes activos en la consecución de los objetivos. 
DIRECCIONAMIENTO ESTRATÉGICO EN SERVICIOS GERONTOLÓGICOS. LA PERCEPCIÓN DE LOS DIRECTIVOS EN TABASCO.

La utilización de la valoración geriátrica integral como herramienta para identificar expectativas y demandas de atención, también se observó en las instituciones públicas. Esto demuestra su utilidad para obtener información sobre el estado del adulto mayor y formular estrategias de atención coherentes con sus características particulares. Además, permite la racionalización de los recursos, la disminución de los costos, y mejorar la calidad de la atención (D'Hyver de las Deses, 2017).

Dos directivos de instituciones públicas afirmaron que su estrategia utilizada era la observación participante durante la convivencia con los usuarios. Esta estrategia, fomenta la contribución directa de las personas mayores en la definición de los objetivos de atención de la organización, al tiempo que promueve su participación social al ofrecer un espacio de reconocimiento personal (Gallardo, 2016).

Las estrategias de los directivos informantes para identificar las expectativas y necesidades de atención gerontológica de los usuarios, se resumen en la Tabla 7.

\section{Tabla 7}

Sensibilidad ante las expectativas y necesidades de los usuarios percibida por los directivos

\begin{tabular}{ll}
\hline Categoría Discurso &
\end{tabular}

Sensibilidad a las expectativas y necesidades de los usuarios

\begin{abstract}
“...teníamos un programa basado en un modelo de logro de metas. Por ejemplo, pon tú que un paciente no contenía el esfínter, ingresaba sin contener esfínteres; mi tarea, la tarea del personal, y a lo que yo me comprometía con la familia, era que, por ejemplo, en un mes, este paciente ya no iba a necesitar protectores o que ya iba a contener esfínteres... es muy importante comunicarle al paciente, al adulto mayor cuáles son las metas, qué es lo que quieres lograr y qué es lo que quieres hacer. En los casos en que ya hay un proceso demencial establecido de todas maneras se les tiene que explicar, probablemente no se acuerden al rato, se les olvida o tienen momentos de rebeldía, se enojan contigo, pero terminan haciéndolo, que esa es la parte más importante. Ven que los demás lo hacen y ellos también quieren hacerlo, y eso hace que mejore su calidad de vida. De alguna forma creo que esa es la clave, el buscar las estrategias, pero siempre comunicándolo tanto a ellos como a los familiares". SM-D01.

"Sí, definitivamente se lo tenías que preguntar, platicabas con ellos, y ellos plenamente te lo decían. Pero tú podías hacer un diagnóstico al observar cómo comían, cómo caminaban, cómo se desenvolvían. Día a día podrías observar el deterioro o el avance que tenía en ciertas áreas, entonces lo que uno tiene que hacer es eso, enrolarse, tratar de trabajar en conjunto con todas las áreas para poder observar esas situaciones". SM-D02.
\end{abstract}

"A los residentes los evaluamos en todos los aspectos: los evaluamos emocionalmente, los evaluamos en la satisfacción del servicio, los evaluamos mentalmente, físicamente, nutricionalmente, médicamente, rehabilitatoria... o sea, en todas las escalas de evaluación geriátrica que se manejan, las implementamos y se las aplicamos a todos ellos. SM-D03.

"Preguntando. No hay mejor encuesta, no hay mejor obtención de información si no es que tus oídos estén atentos a escuchar. Y así era". SM-D04.

Fuente: Elaboración propia a partir del análisis de los discursos emitidos por los directivos de instituciones gerontológicas entrevistados. 
DIRECCIONAMIENTO ESTRATÉGICO EN SERVICIOS GERONTOLÓGICOS. LA PERCEPCIÓN DE LOS DIRECTIVOS EN TABASCO.

\section{CONCLUSIONES}

El envejecimiento es un desafío demográfico que se incrementará en el futuro. Para los próximos años, se prevé que la mayor parte de las demandas de atención provenga de este segmento poblacional, por lo que la toma de decisiones para el diseño, implementación y evaluación de las estrategias de los centros de atención gerontológica debe estar fundamentada en un análisis minucioso de los recursos disponibles, la magnitud de los problemas y los beneficios potenciales, que a su vez deberán complementarse con las expectativas legítimas de la población y su contexto.

En este sentido, el estudio realizado sobre el direccionamiento estratégico permitió la identificación de ciertos elementos que contribuyen al funcionamiento de las instituciones gerontológicas en Tabasco; en particular, el reconocimiento del objetivo fundamental de la atención integral del adulto mayor, el pragmatismo de los centros privados en contraste a la complejidad organizativa de las instituciones pública, y al enfoque en los procesos directivos.

Dado que el estado de emergencia sanitaria causado por COVID-19, fue un factor condicionante en el desarrollo del estudio (distanciamiento social obligatorio como medida sanitaria para la protección de la comunidad), se recomienda profundizar en esta temática, cuando las características sanitarias lo permitan.

\section{REFERENCIAS BIBLIOGRÁFICAS}

Assisted Living Workgroup. (2003). Assuring quality in assisted living: Guidelines for Federal and state policy, state regulation, and operations. Recuperado 07 de septiembre 2020, de https://www.huduser.gov/portal/sites/default/files/pdf/Assuring-Quality-in-Assisted-LivingGuidelines.pdf

Consejo Nacional de Población. (2019). Colección. Proyecciones de la población de México y de las entidades federativas 2016-2050. República Mexicana. (1 ${ }^{\mathrm{a}}$ ed.). Recuperado 27 agosto 2020, de www.gob.mx/cms/uploads/attachment/file/487366/33_RMEX.pdf

D'Hyver de las Deses, C. (2017) Valoración geriátrica integral. Rev. Fac. Med. (Méx.), 60(3), 3854. Recuperado 09 de septiembre 2020, de http://www.scielo.org.mx/scielo.php?script=sci_arttext\&pid=S0026$17422017000300038 \&$ Ing=es. 
DIRECCIONAMIENTO ESTRATÉGICO EN SERVICIOS GERONTOLÓGICOS. LA PERCEPCIÓN DE LOS DIRECTIVOS EN TABASCO.

Gálvez, M. et al. (2016). Utilidad de la valoración geriátrica integral en la evaluación de la salud del adulto mayor. Rev. perú. med. exp. Salud pública, 33(2), 321-327. Recuperado 09 de septiembre 2020, de http://dx.doi.org/10.17843/rpmesp.2016.332.2204.

Gallardo, L, et al. (2016). Asociación entre envejecimiento exitoso y participación social en personas mayores chilenas. Gerokomos 27(3), 104-108. Recuperado 09 de septiembre 2020, de http://scielo.isciii.es/scielo.php?script=sci_arttext\&pid=S1134928X2016000300004\&lng=es.

García, M. (2013). Estructuras administrativas actuales en las residencias de los adultos mayores y su frente en la toma de decisiones para el bienestar de los usuarios en la ciudad de Cali. Universidad Autónoma de Occidente. Recuperado 07 de septiembre 2020, de https://red.uao.edu.co/bitstream/10614/5221/1/TAD01605.pdf

Góngora, J. (2018). Reglas de operación de la casa del Árbol, Residencia del anciano para el ejercicio fiscal. (1ra ed.). Recuperado 28 de agosto 2020, de http:transparencia.tabasco.gob.mx/media/DIF/2018/1/442045.pdf

Gutiérrez, S. A. (2016). Direccionamiento estratégico en Instituciones de Salud de primer nivel de atención en Bogotá a través del Balanced Scorecard. Universidad Nacional de Colombia. $\begin{array}{lllll}\text { Recuperado } & 27 & \text { de } & \text { agosto } & \text { de }\end{array}$ http://bdigital.unal.edu.co/57413/1/1032443857.2017.pdf

Huenchuan, S., Rodríguez, R. I. (2015). Necesidades de Cuidado de las Personas Mayores en la Ciudad de México: diagnóstico y lineamientos de política. CEPAL. Recuperado 07 de septiembre 2020 https://repositorio.cepal.org/bitstream/handle/11362/38879/1/S1500754_es.pdf

Instituto Nacional de las Personas Adultas Mayores. (2012). Modelos de Atención Gerontológica. $\begin{array}{lllll}\text { Recuperado } 28 & \text { de } & \text { agosto } & \end{array}$ http://www.inapam.gob.mx/work/models/INAPAM/Resource/Documentos_Inicio/Libro_Mod elos_de_Atencion_Gerontologica_\%28web\%29.pdf

Procuraduría Federal del Consumidor. (2018). Asilos. Una alternativa para el cuidado y atención de los adultos mayores. Recuperado 27 de agosto 2020, de https://www.gob.mx/profeco/documentos/asilos-una-alternativa-para-el-cuidado-yatencion-de-los-adultos-mayores?state=published

Secretaría de Bienestar. (2019). Programa Anual de Trabajo 2019 del Instituto Nacional de las Personas Adultas Mayores. Recuperado 28 de agosto 2020, de http://www.bienestar.gob.mx/work/models/INAPAM/Transparencia/PlanesProlnf/PAT2019. pdf

Trujillo, F. (2006). Direccionamiento estratégico CMAPS. Proc. of the Second Int. Conference on Concept Mapping. San José, Costa Rica. Recuperado 27 agosto 2020, de http://cmc.ihmc.us/cmc2006Papers/cmc2006-p69.pdf

RODRÍGUEZ-SANTOS R. A., PRIEGO-ÁLVAREZ H. R., LÓPEZ-SOLORSANO S., CALDELAS-GARCÍA D. 